\section{SAT0283 ANTIBODIES TO CARBAMYLATED VIMENTIN IN PATIENTS WITH SYSTEMC LUPUS ERYTHEMATOSUS ARE ASSOCIATED WITH RENAL INVOLVENMENT}

F.R. Spinelli, S. Truglia, T. Colasanti, A. Pecani, F. Ceccarelli, F. Miranda, R. Mancini, C. Perricone, M. Pendolino, C. Alessandri, G. Valesini, F. Conti. Dipartimento di Medicina Interna e Specialità Mediche - Reumatologia, Sapienza Università di Roma, Rome, Italy

Background: Vimentin is a cytoskeleton protein of the intermediate filaments family, expressed by mesenchymal cells including vascular endothelial cells and renal tubular cells. Vimentin has been recently proposed as a target of the in situ immune response in lupus nephritis. Antibodies to vimentin have been described in $10-53 \%$ of patients with Systemic Lupus Erythematosus (SLE) and account for a fibrous pattern of cytoplasmic immunofluorescence. Post-translational modifications increase the immunogenicity of vimentin, as demonstrated by the detection of anti-modified vimentin antibodies in patients with rheumatoid arthritis. Carbamylation is a non-enzymatic post-translational modification consisting in the addition of a cyanate group on lysine and arginine residues; since carbamylation is time dependent, structural proteins with a slow turnover are more likely to be carbamylated. The role of carbamylated vimentin as an antigenic target in SLE has not been yet evaluated.

Objectives: Aims of the study were to assess the prevalence of anti-carbamylated vimentin in a cohort of SLE patients and to evaluate the possible associations with clinical and serological feature of the disease.

Methods: Patients with SLE classified according to 1987 ACR criteria were enrolled. Clinical features, autoantibodies profile and disease activity - as measured by SLE Disease Activity Index 2000 (SLEDAI 2K) - were collected. Sera obtained from each patient were tested for anti-carbamylated vimentin by a home-made enzyme-linked immunoassay. Data were expressed as mean \pm standard deviation or median (interquartile range) when appropriate. To investigate difference in anti-carbamylated vimentin prevalence and anti-carbamylated vimentin serum levels Mann-Whitney and Chi square test were applied. $\mathrm{P}$ value $<0.05$ was considered statistically significant.

Results: We enrolled 109 SLE patients (102F:7M, mean age 39.4 \pm 12.6 years, mean disease duration $10.5 \pm 9.5$ years, mean SLEDAI $2 \mathrm{~K} 5 \pm 5.5$ ).

Overall, 30 out of 109 patients (27.5\%) were positive for anti-carbamylated vimentin. According to the clinical features, the prevalence of anti-carbamylated vimentin was significantly higher in patients with lupus nephritis (18/44) compared to those without renal involvement $(12 / 66)(41.8 \%$ vs $18.2 \%, p=0.006)$; moreover, anti-carbamylated vimentin serum levels were significantly higher in patients with lupus nephritis [2561 (1783) OD] compared to those without [1970 (1123) OD; $\mathrm{p}=0.0178]$. We didn't find any difference in prevalence or titre of anti-carbamylated vimentin according to presence/absence of other clinical manifestations (musculoskeletal, muco-cutaneous, hematologic, neuropsychiatric) or serology (low complement, anti-dsDNA). Moreover, we did not find any correlation between anti-carbamylated vimentin serum levels and SLEDAI $2 \mathrm{~K}$.

Conclusions: Higher prevalence and serum levels of anti-carbamylated vimentin antibodies in patients with lupus nephritis confirm the role of vimentin as a target of the immune response in patients with glomerulonephritis and suggest their possible role as a biomarker of kidney involvement in SLE patients.

Disclosure of Interest: None declared

DOI: 10.1136/annrheumdis-2017-eular.5819

\section{SAT0284 ASSOCIATION OF PATHOGENIC AND REGULATORY B-CELL SUBSETS WITH CLINICAL AND HISTOLOGICAL FEATURES IN PRIMARY SJÖGREN'S SYNDROME}

F. Carubbi $^{1,2}$, A. Alunno $^{3}$, P. Cipriani ${ }^{1}$, P. Di Benedetto ${ }^{1}$, V. Liakouli ${ }^{1}$,

P. Ruscitti ${ }^{1}$, O. Berardicurti ${ }^{1}$, S. Di Bartolomeo ${ }^{1}$, R. Gerli ${ }^{3}$, R. Giacomelli ${ }^{1}$.

${ }^{1}$ Department of Biotechnological and Applied Clinical Science, Rheumatology

Unit, School of Medicine, University of L'Aquila; ${ }^{2}$ Department of Medicine, ASL 1

Avezzano-Sulmona-L'Aquila, L'Aquila; ${ }^{3}$ Department of Medicine, Rheumatology

Unit, University of Perugia, Perugia, Italy

Background: Several data pointed out that B-cells play a central role in the development, maintenance and progression of primary Sjögren's syndrome (pSS) B-lymphocyte hyperactivity, salivary gland (SG) infiltration, and the development of B-cell follicles containing germinal center (GC)-like structures, represent hallmarks of the disease. On this basis, B-cell depleting therapy with anti-CD20 monoclonal antibody rituximab (RTX) has been employed in pSS showing promising results. However, currently available data do not allow to draw definitive conclusion on its efficacy.

Objectives: Aim of the study was to assess mature and regulatory B cell subsets in patients with pSS and explore possible associations with clinical and histological features and their possible modulation by RTX treatment.

Methods: We evaluated by flow cytometry peripheral blood (PB) B-cell subsets (mature B cells: Bm1, Bm2, Bm2', Bm3, Bm4, early(e)Bm5, Bm5; IL-10-producing B-cells), in 17 pSS patients and in 10 healthy donors (HD). Nine pSS patients received a course of RTX (1000 mg at days 1 and 15) and PB B-cell subsets were also evaluated after 3 (T3) and 6 (T6) months and compared to baseline values (T0).

Results: We confirmed that the percentage of $\mathrm{Bm} 1$, eBm5 and $\mathrm{Bm} 5$ was lower and that of Bm2, Bm2' and Bm3+Bm4 was higher in pSS patients compared to
$H D$ (all $p<0.05)$. When we divided patients according to the ESSDAI score $(<5$ or $\geq 5$ ) or to SG-GC (presence/absence), we observed for the first time that the percentage of Bm2' cells, also called GC founders, was higher in patients with ESSDAI $\geq 5 \quad(p=0.02)$ compared to those with lower ESSDAl score and in pSS patients with $G C(p=0.02)$ compared to those without GC. Although we observed a reduction of all B-cells subsets at T3 and T6 after RTX treatment, the proportion of $\mathrm{Bm} 1, \mathrm{Bm} 3+\mathrm{Bm} 4, \mathrm{eBm} 5$ and $\mathrm{Bm} 5$ persisted lower and the percentage of $\mathrm{Bm} 2$ and $B m 2$ ' persisted higher in pSS patients $(p<0.05)$ compared to HD. No differences regarding IL-10-producing $B$-cells was obeserved between pSS patients and HD. However, the percentage of IL-10-producing B-cells was higher in patients with $E S S D A I \geq 5$ compared to patients with $\operatorname{ESSDAI}<5 \quad(p=0.02)$.

Conclusions: We demonstrated for the first time that a subset of circulating mature B-cells, Bm2', also called GC founders, is strongly associated with higher disease activity and with the presence of SG-GC. Moreover, a higher ESSDAI is associated with higher percentage of circulating IL-10-producing B-cells Pathogenic B-cell hyperactivity is a hallmark of pSS, however B-cells are also a source of inhibitory cytokines such as IL-10 and TGF-beta and the increase of IL-10 producing $B$ cells that we observed in active pSS patients may suggest an ongoing compensatory mechanism to counteract chronic inflammation. Therefore, the design of novel $B$ cell targeted therapies should ensure that only pathogenic $B$ cells are depleted and regulatory B cell subsets are not hampered while, ideally, potentiated.

References:

[1] Binard A et al. Ann Rheum Dis 2009:68:1447-1452.

[2] Carubbi F et al. Arthritis Res Ther 2013;15:R172.

[3] Carubbi F et al. Lupus 2014; 23:1337-49.

[4] Kroese FG, et al. B-cell hyperactivity in primary Sjögren's syndrome. Expert Rev Clin Immunol. 2014;10:483-99.

Disclosure of Interest: None declared

DOI: 10.1136/annrheumdis-2017-eular.4407

\section{SAT0285 THE PHYSICAL PERFORMANCE OF SYSTEMIC LUPUS ERYTHEMATOSUS PATIENTS WITH LOW DISEASE ACTIVITY: COULD THE CARDIOPULMONARY EXERCISE TESTING REFINE ITS ASSESSMENT?}

G. Gusetu ${ }^{1}$, A. Mociran ${ }^{2}$, C. Pamfil ${ }^{3}$, L. Damian ${ }^{2}$, D. Pop ${ }^{1}$, D. Zdrenghea ${ }^{1}$, S. Rednic ${ }^{3}$. ${ }^{1}$ Cardiology, University of Medicine and Pharmacy; ${ }^{2}$ Rheumatology, County Emergency Hospital; ${ }^{3}$ Rheumatology, University of Medicine and Pharmacy, Cluj-Napoca, Romania

Background: Even during remission, the systemic lupus erythematosus (SLE) patients have reduced exercise performance and this contributes to impairment of their quality of life ${ }^{1}$. Several causes as depression and deconditioning, arthritis, anemia, cardiovascular and respiratory involvement are widely accepted; however the weight of each factor is less known in particular individuals.

Objectives: Our study aimed to assess through cardiopulmonary exercise testing (CPX) the exercise performance of a SLE cohort and to establish its main determinants.

Methods: Thirty-one SLE patients with low disease activity underwent a CPX on cycle ergometer; the main metabolic parameters and standard 12-leads ECG were recorded; before exercise testing, a cardiac Doppler ultrasound was performed. The patient's characteristics, cumulative organ damage and laboratory data were retrieved by medical chart review. The control group consisted of 25 age and sex-matched healthy, non-trained individuals.

Results: Within the study group, 28 (90.3\%) were female, the mean age was $42.7 \pm 10.6$ years and disease median duration 7.9 years. The aerobic performance was decreased by $16.2 \%$ (17.6 vs $21.36 \mathrm{ml} / \mathrm{kg} / \mathrm{min}, \mathrm{p}=0.022$ ); the main disease characteristics which correlated with maximum oxygen uptake (VO2Mx) were anemia $(p=0.035)$, renal involvement $(p=0.05)$ and antiphospholipid syndrome (APS) $(p=0.042)$ but not disease duration, cumulative damage or the immunological tests (hypocomplementemia, anti-Ro, anti-Sm, anti-dsDNA, AAN or APL antibodies).

One quarter of patients did not reach the ventilatory anaerobic threshold (VAT, expressed as a percentage of calculated VO2Mx), mainly due to musculoskeletal pain (5 patients), dyspnea (2 patients with history of pulmonary embolism) and sudden rise in blood pressure (1 patient). Among the rest of them (23 patients, $74 \%)$, the VAT was at the lower limit of normal range $(41.03 \%$ vs $54.0 \%$ for controls, $\mathrm{p}=0.014$ ) corresponding to a "training reserve" of $31 \%$. Of particular importance from this point of view were the criteria for test termination: dyspnea in 4 patients ( 1 with anemia, 1 with pulmonary fibrosis and 2 by hyperventilation proved by mean VE/VCO2 $=41.5$ ), fatigue ( 9 patients) and arthralgia ( 7 patients). Notably, among patients with established diagnosis of mild pulmonary fibrosis, COPD, ischemic or valvular heart disease, not dyspnea but arthralgia or fatigue was the reason for test termination, even if the VO2Mx was lower than recorded in the rest of the group ( $15.8 \mathrm{vs} 19.2 \mathrm{ml} / \mathrm{kg} / \mathrm{min}, \mathrm{p}=0.037$ ).

Conclusions: The exercise aerobic capacity of SLE patients is diminished and correlates with anemia, renal involvement and with hystory of pulmonary embolism. Surprisingly, even in patients with mild cardiovascular or respiratory involvement, the decreasing of exercise performance is limited mainly from musculoskeletal symptoms and from deconditioning.

References:

[1] Carvalho MR, Sato EI, Tebexreni AS, et al. Effects of supervised cardiovascular 